\title{
The Treatment of Multiple Sclerosis with Inosine
}

\author{
Clyde E. Markowitz, M.D., ${ }^{1}$ Sergei Spitsin, Ph.D., ${ }^{2}$ Vanessa Zimmerman, M.S.N., ${ }^{1}$ Dina Jacobs, M.D., ${ }^{1}$ \\ Jayaram K. Udupa, M.D., ${ }^{3}$ D. Craig Hooper, Ph.D., ${ }^{2}$ and Hilary Koprowski, M.D. ${ }^{2}$
}

\begin{abstract}
Objective: The objective of this study is to evaluate the safety and tolerability of inosine in patients with relapsing-remitting multiple sclerosis (RRMS). The secondary objectives are to assess the effects of inosine administration on serum urate (UA) levels, the progression of neurologic disability, the cumulative number of new, active lesions on magnetic resonance imaging (MRI), and changes in serum levels for markers of inflammation. Design: Oral administration of inosine was used to raise serum levels of the natural peroxynitrite scavenger UA in 16 patients with RRMS during a 1-year randomized, double-blind trial.

Outcome measures: The endpoints studied were relapse rate, disability assessed by the Kurtzke Expanded Disability Status Scale (EDSS), MRI, and analysis of serum levels of nitrotyrosine, and oxidative and proinflammatory makers.

Results: Increased serum UA levels correlated with a significant decrease in the number of gadoliniumenhanced lesions and improved EDSS. A number of MRI intensity-based parameters were altered by inosine treatment, in certain cases correlating with changes in serum UA levels. In a patient with low serum UA and high lesion activity, raising UA levels by inosine treatment decreased serum nitrotyrosine while increasing the ratio of Th2 to Th1 cytokines in circulating cells. The only side-effect correlated with inosine treatment was kidney stone formation in $4 / 16$ subjects.

Conclusions: These data suggest that the use of inosine to raise serum UA levels may have benefits for at least some MS patients. The effect of this treatment is likely to be a consequence of inactivation of peroxynitritedependent free radicals. Close monitoring of serum UA levels as well as other measures are required to avoid the potential development of kidney stones.
\end{abstract}

\section{Introduction}

$\mathbf{M}$ ULTIPLE SCLEROSIS (MS) is one of the most common neurologic diseases of young adults, accounting for more disability, treatment costs, and lost income than any other neurologic disease in this age group in Western Europe and in North America. ${ }^{1,2}$ A principal anatomic feature of MS is the development of inflammatory lesions, predominantly in the white matter of central nervous system (CNS) tissues. ${ }^{2-4}$ Magnetic resonance imaging (MRI) is widely used as a diagnostic tool in MS and is beginning to be used to monitor disease progression by examining different MRI parameters., New MRI methods and analytical techniques are being developed to provide additional clinically relevant information.
Active MS plaques generally contain inflammatory cells that express intracellular inducible nitric oxide synthase (iNOS) and produce peroxynitrite-dependent radicals, which can be detected by their nitration of tyrosine residues. ${ }^{7-9}$ The contribution of peroxynitrite-dependent radicals to CNS lesion formation has been extensively studied in animal models, where the therapeutic effects of the natural peroxynitritedependent radical scavenger uric acid (UA) has been demonstrated. ${ }^{10,11}$ These findings are significant for patients with MS who often have lower serum UA levels than normal individuals. ${ }^{12}$ In addition, an inverse correlation between the occurrence of MS and serum UA levels has been demonstrated. ${ }^{12}$

Based on these observations, a pilot study was conducted in patients with secondary-progressive MS (SPMS) to test

\footnotetext{
${ }^{1}$ Neurology Department, University of Pennsylvania, Philadelphia, PA.

${ }^{2}$ Biotechnology Foundation Laboratories at Thomas Jefferson University, Philadelphia, PA.

${ }^{3}$ Department of Radiology, University of Pennsylvania, Philadelphia, PA.
} 
whether blood urate levels could be raised by oral administration of UA to patients with MS. ${ }^{13}$ This proved to be unsuccessful, likely due to degradation of UA by gastrointestinal bacteria. ${ }^{13}$ A follow-up study was performed demonstrating that the UA precursor inosine could be successfully used to raise serum UA levels. ${ }^{13}$ Inosine is a dietary supplement available in most health food stores and is taken by some athletes to boost performance, although scientific studies have failed to support this belief. ${ }^{14-16}$ No inosine-related sideeffects were reported in the MS pilot trial, but no benefit in SPMS was apparent, likely due to low disease activity. ${ }^{13}$ Subsequently, it has been reported that 32 patients with MS receiving the relatively low dose of $1-2 \mathrm{~g}$ of inosine per day for a period of approximately 3 years had significantly lower relapse rates and smaller increases in Kurtzke Expanded Disability Status Scale (EDSS) score than 32 nontreated controls. $^{17}$

The current study was designed to evaluate further the safety and tolerability of inosine in patients diagnosed with relapsing-remitting multiple sclerosis (RRMS) as well as to provide a preliminary efficacy analysis.

\section{Methods}

\section{Patient population}

Sixteen (16) patients at the University of Pennsylvania MS Center were selected for the study based upon the criteria reported in Table 1, including diagnosis of RRMS based on the McDonald criteria, an EDSS $\leq 5$, and serum UA levels of $<5 \mathrm{mg} / \mathrm{dL}$. Exclusion criteria included treatment with interferons, glatiramer acetate, or other immune-modifying reagents within the previous 3 months, or corticosteroids within 1 month of the initial baseline MRI assessment.

\section{Study design}

The study was randomized, double-blinded, of 1-year duration consisting of two arms: (1) a placebo-controlled crossover study consisting of 6 months placebo then 6 months of inosine, and (2) inosine treatment for 12 months following baseline assessment. The protocol was approved by regulatory agencies and the institutional review boards of Thomas Jefferson University (TJU) and University of Pennsylvania, and the trial was carried in accordance with accepted guidelines. ${ }^{18}$ (Trial registration: clinicaltrials.gov, identifier: NCT00067327). Subject recruitment and consent was carried out by investigators at the University of Pennsylvania, and randomization was performed by TJU investigators using the stratification method. Capsules were provided to patients in containers labeled with a random four-digit lot number. The sequence of capsule content was concealed from the subjects and the study team at the University of Pennsylvania. Target recruitment for this pilot study was 20 subjects.

\section{Clinical assessments}

Patients were evaluated monthly by MRI, blood tests, and adverse events. Neurologic examinations (EDSS) were performed every 3 months and physical examinations every 6 months. Patients, examining and treating neurologists, study coordinators, and MRI analysis personnel were all blinded to the treatment. Inosine dose adjustments were based on blood urate levels and made by unblinded investigator at TJU so as to not unblind the study team. Intravenous (IV) methylprednisolone $1000 \mathrm{mg} /$ day for 3 days was allowed for the treatment of relapses.

\section{Treatment}

Capsules with identical appearance containing either $500 \mathrm{mg}$ of inosine or $500 \mathrm{mg}$ of fructose (placebo) were both prepared by JoMar Laboratories (Campbell, CA). To minimize the possibility that differences between the capsules could be differentiated by taste, subjects were instructed to swallow the capsules whole. The target serum UA level was 6-9 mg/dL. To achieve this level, the inosine dosage was initially $1-2 \mathrm{~g} /$ day, increasing by $0.5 \mathrm{~g} /$ day at biweekly intervals. For most patients, the inosine dose required to maintain target UA levels was between 2 and $3 \mathrm{~g} /$ day. If blood UA levels exceeded $9 \mathrm{mg} / \mathrm{dL}$, the inosine dose was

Table 1. Patient Characteristics

\begin{tabular}{|c|c|c|c|c|c|c|}
\hline Patient & Age & Ethnicity & $\begin{array}{c}\text { Years since diagnosis } \\
\text { with MS }\end{array}$ & Sex & $\begin{array}{l}\text { Weight } \\
(\mathrm{kg})\end{array}$ & $\begin{array}{l}\text { Baseline } \\
\text { EDSS }\end{array}$ \\
\hline 1 & 54 & African American & 1 & $\mathrm{~F}$ & 60 & 1.5 \\
\hline 2 & 30 & White & 3 & $\mathrm{~F}$ & 101 & 2.0 \\
\hline 3 & 32 & White & 1 & $\mathrm{~F}$ & 94 & 2.0 \\
\hline 4 & 22 & White & 1 month & $\mathrm{F}$ & 70 & 1.5 \\
\hline 5 & 45 & White & 3 months & M & 61 & 4.0 \\
\hline 6 & 27 & White & 6 & $\mathrm{~F}$ & 65 & 2.0 \\
\hline 7 & 39 & White & 3 months & F & 87 & 2.0 \\
\hline 8 & 40 & White & 6 & $\mathrm{~F}$ & 60 & 2.5 \\
\hline 9 & 55 & White & 6 months & $\mathrm{F}$ & 72 & 2.0 \\
\hline 10 & 49 & White & 1 month & F & 64 & 2.5 \\
\hline 11 & 50 & White & 13 & $\mathrm{~F}$ & 89 & 3.5 \\
\hline 12 & 54 & White & 4 & $\mathrm{~F}$ & 73 & 3.0 \\
\hline 13 & 29 & White & 8 & $\mathrm{~F}$ & 74 & 2.0 \\
\hline 14 & 54 & African American-European Jewish & 3 & $\mathrm{~F}$ & 77 & 2.5 \\
\hline 15 & 59 & African American & 11 & $\mathrm{~F}$ & 95 & 5.0 \\
\hline 16 & 42 & White & 12 & $\mathrm{~F}$ & 79 & 3.5 \\
\hline
\end{tabular}

MS, multiple sclerosis; EDSS, Kurtzke Expanded Disability Status Scale. 
reduced. Blood work for complete blood count (CBC), blood urea nitrogen (BUN), creatinine, and UA levels was initially conducted biweekly and later at monthly intervals. Following the development of kidney stones in a few patients, detailed dietary guidelines were developed for the last 5 patients recruited. These guidelines included adequate fluid intake (6-8 glasses of water daily), a low purine and low oxalate diet, limited alcohol (especially beer), and dietary calcium intake (1000-1300 mg daily), as opposed to oral supplements.

\section{$M R I$ and image analysis}

MRI scans were performed at baseline and at monthly intervals using a 1.5-T Siemens scanner. MRI scans were analyzed in a blinded fashion at the University of Pennsylvania using 3DVIEWNIX software and published approaches. ${ }^{19-23}$ Different tissue regions were delineated in the images as follows: In T2 and proton density (PD) images: brain parenchyma (BP), gray matter (GM), white matter (WM), and MS lesions (LS). The WM region was further divided into pure WM (PWM) and dirty or diseased WM (DWM) regions. Parameters were divided into two groups: (1) morphological (volumes of BP, GM, WM, PWM, DWM, T2 lesions, T1E lesions); and (2) intensity-based (for each tissue region intensity histogram in each of T2, PD, T1E, and magnetization transfer ratio [MTR] images was computed).

Changes in MR parameter values were tested from baseline to last month of treatment for both placebo and inosine groups. Baseline was considered as the month preceding the start of treatment.

\section{Quantitative reverse transcription-polymerase chain reaction}

Blood was collected into heparinized tubes and white blood cells were isolated by density centrifugation on Ficoll-Paque (Amersham Biosciences, Uppsala, Sweden). All details of RNA isolation, cDNA synthesis, and quantitative polymerase chain reaction using the Bio-Rad iCycler iQ Real Time Detection System (Hercules, CA) are described. ${ }^{24}$ Probes and primers for human gene-specific mRNAs are presented in Table 2 and primers for the corresponding standards in Table 3.

\section{Serum analyses}

Serum levels of cytokines, nitrotyrosine, and oxidative markers were determined using the following kits according to the manufacturers' recommendations: 8-isoprostane, nitrotyrosine, and 8-hydroxy-2'-deoxyguanosine kits from (Oxis International, Foster City, CA), human interleukin (IL)-10 and IL-13 kits (Pelikine Research Diagnostics, Flanders, NJ), human granulocyte-macrophage colony-stimulating factor enzymelinked immunosorbent assay (ELISA) kit (BD Pharmingen, San Jose, CA), human tumor necrosis factor- $\alpha$ and human interferon- $\gamma$ ELISA kits (Research Diagnostics Inc., Concord, MA), soluble intercellular adhesion molecule-1 (sICAM-1) ELISA kit ( + D Systems, Minneapolis, MN). Lipid peroxidation was assessed as malondialdehyde (MDA) formation in sera by thiobarbituric acid reaction as described. ${ }^{25}$

\section{Statistical analysis}

Evaluation of the significance of differences between parameters was performed using analysis of variance or

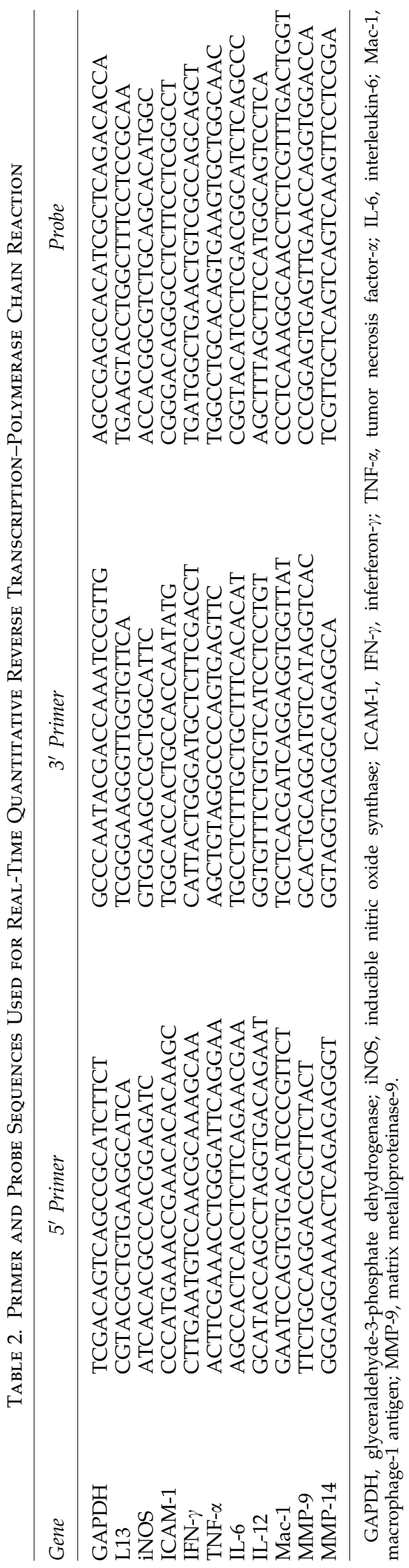


Table 3. Primer Sequences Used to Synthesize cDNA Standards for Real-Time Quantitative Reverse Transcription-Polymerase Chain Reaction

\begin{tabular}{lll}
\hline Gene & \multicolumn{1}{c}{$3^{\prime}$ Primer } & $5^{\prime}$ Primer \\
\hline GAPDH & TCGACAGTCAGCCGCATCTTCT & ATGGCATGGACTGTGGTCAT \\
L13 & CCATCGTGGCTAAACAGGTA & TTTATTGGGCTCAGACCAGG \\
iNOS & AGAGTACAGCAAGTGGAAGT & TCCTGAACATAGACCTTGGG \\
ICAM-1 & CGAGGTGACCGTGAATGTG & AGTCTTGCTCCTTCCTCTTG \\
IFN- $\gamma$ & GTGTGGAGACCATCAAGGAA & ACCCATGGGATCTTGCTTAG \\
TNF- $\alpha$ & GCCCGACTATCTCGACTT & GCTACATGGGAACAGCCTAT \\
IL-6 & TGAACTCCTTCTCCACAAGC & CCATGCTACATTTGCCGAAG \\
IL-12 & GACCAGCCTGGGAAACATAA & GCATCTGTCTGCTTCTCACA \\
Mac-1 & GCCGGTGAAATATGCTGTCTAC 9 & TCCACTTTGGTCTCCGTCT \\
MMP-14 & AGGCGTCTGGACAAGCTG & CCAAGTTTATTAGAAACACTCCAAC \\
\hline
\end{tabular}

GAPDH, glyceraldehyde-3-phosphate dehydrogenase; iNOS, inducible nitric oxide synthase; ICAM-1, intercellular adhesion molecule-1; IFN- $\gamma$, inferferon- $\gamma$; TNF- $\alpha$, tumor necrosis factor- $\alpha$; IL-6, interleukin-6; Mac- 1 , macrophage- 1 antigen; MMP-9, matrix metalloproteinase- 9 .

Student's t-test, Wilcoxon rank sum test, Pearson correlation analysis, and Fisher's exact test as indicated. In all cases, $p<0.05$ was considered significant.

\section{Results}

\section{Safety profile and effect of inosine on serum UA levels}

Subjects were recruited to the study between 2002 and 2006 with follow-up observations continuing until early 2008. Of 16 patients recruited for the study, 12 completed the study. Three (3) patients were removed due to the formation of kidney stones. One (1) patient withdrew because of unwillingness to continue with the study protocol. Average serum UA levels were increased from $4.2 \pm 0.8$ to $7.2 \pm 1.8 \mathrm{mg} / \mathrm{dL}$ by inosine treatment. Target UA levels of 6-9 mg/dL were consistently maintained in 2 patients, while in the remaining 10 patients, levels below and in excess of the target occurred between $15 \%$ and $65 \%$ of the time during the inosine phase. In 3 cases, low levels were confirmed to be the result of poor subject compliance. No changes in other monitored blood parameters including CBC, BUN, creatinine, aspartate aminotransferase, and alanine aminotransferase were associated with inosine treatment. Four (4) of the first 11 patients recruited for the trial developed kidney stones, which were treated with conservative measures including termination of inosine administration, improved hydration, and pain medication. In all patients in whom inosine treatment was stopped, serum UA levels returned to pretrial levels within 3-5 days and no re-occurrence of kidney stones was reported. This included a subject who resumed inosine treatment 2 months after developing a kidney stone. In 1 patient who developed kidney stones, an ultrasound scan revealed the presence of kidney cancer in the other kidney, which was successfully treated surgically. No kidney stones were reported after the adoption of dietary guidelines.

\section{Clinical outcomes}

In the cross-over arm of the study, average EDSS scores were unchanged during the placebo phase ( $3.1 \pm 1.1$ baseline and $3.1 \pm 1.26$ months later) but improved with inosine treatment (from $3.1 \pm 1.2$ to $2.6 \pm 1.7$ ). In subjects who were placed on inosine at the start of the trial, EDSS scores were improved at both 6 months and 1 year (from $2.1 \pm 0.5$ to $1.9 \pm 0.4$ over the first 6 months and to $1.6 \pm 0.7$ by the end of the year). Data are presented in Figure 1. There were no significant differences in the baseline EDSS between the two groups. Statistically significant decrease in EDSS scores from a baseline to the end of the treatment phase $(p=0.01$, by the Wilcoxon rank sum test) correlated with statistically significant increase in UA levels $(p<0.001)$, measured during the same visits.

The effects of inosine treatment on the frequency of gadolinium-enhanced lesions and clinical exacerbations were examined in several different ways. As noted above, inosine treatment did not always result in target serum UA levels of between 6 and $9 \mathrm{mg} / \mathrm{dL}$. When lesion frequency at baseline and during placebo treatment is compared with all points during inosine treatment, there is no significant effect of treatment. As shown in Table 4, the frequency of clinical exacerbations was reduced in patients with MS with serum UA levels greater than $7.0 \mathrm{mg} / \mathrm{dL}$. At the same time, the

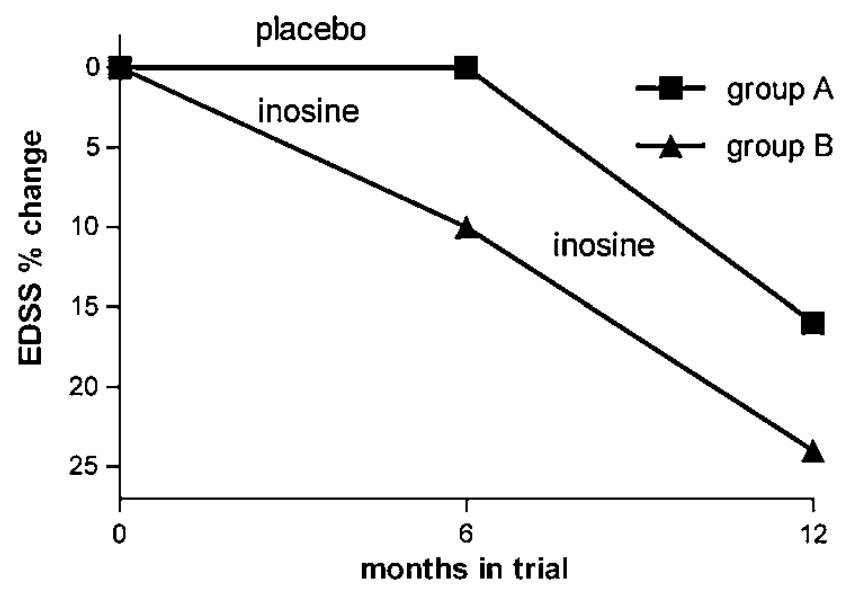

FIG. 1. Improvement of Kurtzke Expanded Disability Status Scale (EDSS) scores during inosine therapy. Graph represents the change in EDSS score as a percent of the baseline assessment for subjects in the two arms of the trial: (1) a crossover study consisting of 6 months of placebo then 6 months of inosine (group A); (2) inosine treatment for 12 months following baseline assessment (group B). 
Table 4. Comparison of Serum Uric Acid (UA) Levels and Disease Activity in Patients with Multiple Sclerosis During Placebo/Inosine Treatment

\begin{tabular}{lccc}
\hline $\begin{array}{l}\text { Serum } \\
\text { UA level }\end{array}$ & $\begin{array}{c}\text { Number of } \\
\text { visits }\end{array}$ & $\begin{array}{c}\text { Total number of } \\
\text { active lesions }\end{array}$ & $\begin{array}{c}\text { Visits with } \\
\text { exacerbations }\end{array}$ \\
\hline $7.0 \mathrm{mg} / \mathrm{dL}$ & 61 & 17 & 1 \\
$<7.0 \mathrm{mg} / \mathrm{dL}$ & 113 & 154 & 6 \\
Total & 174 & 171 & 7 \\
\hline
\end{tabular}

The association between UA level and lesion activity is significant by Fisher's exact test (two-sided) $p<0.001$.

$\mathrm{UA}$, urate.

burden of gadolinium-enhanced lesion during the placebo phase as well as while taking inosine where serum UA levels were less than $7.0 \mathrm{mg} / \mathrm{dL}$ was significantly greater than lesion frequency when serum UA was above $7.0 \mathrm{mg} / \mathrm{dL}$ $(p<0.001$, by Fisher's exact test) (Table 4$)$. Detailed MRI analysis revealed a number of parameters that were significantly different between scans at baseline (Arm 2) or the final placebo assessment (Arm 1) and at the conclusion of inosine treatment. Seven intensity-based parameters showed moderate to strong correlation with the UA level, with an $r$ value of 0.51-0.71 (Table 5). The highest correlations with UA levels were obtained for PD values in the DWM region $(r=-0.71, p=0.001)$. This implies that, as the UA levels are increased in WM regions that may have underlying demyelination but not yet apparent lesions, there may be reversal of this process. Eight (8) standardized PD parameters show moderate to high correlation with the EDSS scores (Table 6), with the highest value of $r=0.75, p=0.001$ observed for PD $\mathrm{BP}$ histogram 75 th percentile. The parameters that correlated with both EDSS and UA levels are PD BP histogram peak location $(r=-0.52, p=0.06$ for UA; $r=0.68, p=0.003$ for EDSS) and PD BP histogram 75th percentile $(r=-0.54$, $p=0.04$ for UA; $r=0.75, p=0.001$ for EDSS).

\section{Inflammatory markers}

Levels of the inflammatory and oxidative markers studied varied considerably during the baseline/placebo and inosine phases of the study such that no significant difference in pooled data could be detected (data not shown). Variability was also extensive within individuals regardless of the stage of the trial. Nevertheless, in the individual in Arm 1 of the study who exhibited the greatest reduction in lesion burden between the placebo and inosine phases (averaging 10.2 \pm 3.8 and $2.0 \pm 1.5$, respectively, $p<0.001$ by the $t$ test), significant

Table 5. Magnetic Resonance Imaging Parameters Showing Significant Correlations WITH URIC ACID LEVELS

\begin{tabular}{lll}
\hline PD BP histogram peak location & $r=-0.52$ & $p=0.06$ \\
PD BP histogram 75th percentile & $r=-0.54$ & $p=0.04$ \\
PD GM histogram peak height & $r=0.51$ & $p=0.06$ \\
T2 GM histogram 25th percentile & $r=0.61$ & $p=0.02$ \\
T2 GM histogram 50th percentile & $r=0.6$ & $p=0.02$ \\
T2 GM histogram 75th percentile & $r=0.59$ & $p=0.03$ \\
PD DWM histogram peak location & $r=-0.71$ & $p=0.001$ \\
\hline
\end{tabular}

$\mathrm{PD}$, proton density; BP, brain parenchyma; GM, gray matter; DWM, diseased white matter.
Table 6. Magnetic Resonance Imaging Parameters Showing Significant Correlations With the KurtzKe Expanded Disability Status Scale Scores

\begin{tabular}{lll}
\hline PD BP histogram peak location & $r=0.68$ & $p=0.003$ \\
PD BP histogram 50th percentile & $r=0.63$ & $p=0.007$ \\
PD BP histogram 75th percentile & $r=0.75$ & $p=0.001$ \\
PD GM histogram peak location & $r=0.71$ & $p=0.001$ \\
PD GM histogram 50th percentile & $r=0.63$ & $p=0.007$ \\
PD GM histogram 75th percentile & $r=0.68$ & $p=0.003$ \\
PD PWM histogram peak height & $r=-0.60$ & $p=0.01$ \\
PD T2-lesion histogram 75th & $r=0.64$ & $p=0.005$ \\
percentile & & \\
\hline
\end{tabular}

$\mathrm{PD}$, proton density; $\mathrm{BP}$, brain parenchyma; GM, gray matter; PWM, pure white matter.

differences were seen in several serum markers including nitrotyrosine and macrophage- 1 antigen (Mac-1) mRNA levels (Table 7).

\section{Discussion}

The main objective this study was to evaluate the safety and tolerability of the use of orally administered inosine to raise serum UA levels in patients diagnosed with MS. While it proved difficult to maintain target serum UA levels in some patients, inosine readily raised UA levels and was well tolerated in the majority of the subjects. The main concern was the development of kidney stones in 4 of the first 11 subjects. Conservative treatment, including immediate cessation of inosine treatment and improved hydration, was sufficient to promote rapid recovery without sequelae. The high incidence of kidney stones was somewhat surprising since we did not observe this complication during a previous trial involving 17 subjects and similar protocol. ${ }^{13}$ The principal difference between the two studies is in the nature of the subjects. Those of the first trial were nonambulatory, nursing home, SPMS patients receiving a defined in-house diet with monitoring for proper hydration and consistent inosine administration. The subjects of the current trial were ambulatory patients residing in their own domiciles without any control or monitoring of inosine compliance, diet, or liquid intake. Although their kidney stones were composed of UA, it was noted that all 4 subjects who developed this complication also took calcium supplements. Two (2) of these patients had very high blood UA levels at the time of kidney stone development (10 and $15 \mathrm{mg} / \mathrm{dL})$, while the other 2 had UA levels within the target range $(6.4$ and $8.4 \mathrm{mg} / \mathrm{dL})$. There are several factors that may contribute to the development of kidney stones during inosine treatment, including transient dehydration or acidification of urine. Another possibility is that some patients may be predisposed to the development of UA kidney stones as a consequence of alterations in the UA excretion/reuptake system responsible for maintaining UA levels. For example, this could occur through mutations in the gene encoding the UA transporter. ${ }^{26}$ We believe that dietary and hydration guidelines are important, and more frequent monitoring of serum UA levels than at monthly intervals would be of benefit, particularly if there is any change in dosage. The kidney cancer that was detected in the opposite kidney from one that developed a stone was an incidental finding picked up on ultrasound. The nephrologist did not feel that this was at all related to the inosine treatment, and the cancer was cured by surgery. 
Table 7. Blood Markers in Patient with the Greatest Response to Inosine Therapy

\begin{tabular}{|c|c|c|c|c|c|}
\hline & $\begin{array}{c}U A \\
(m g / d L)\end{array}$ & $\begin{array}{c}\text { NT by ELISA } \\
(n M)\end{array}$ & $\begin{array}{l}\text { IL-10 by ELISA } \\
\quad(\mathrm{pg} / \mathrm{mL})\end{array}$ & $\begin{array}{c}I L-13 \text { by ELISA } \\
(p g / m L)\end{array}$ & $\begin{array}{c}\text { Mac-1 by PCR } \\
\text { (copy of mRNA per copy } \\
\text { of GAPDH mRNA) }\end{array}$ \\
\hline Placebo & $3.9 \pm 0.7$ & $73 \pm 40$ & $3.1 \pm 3.0$ & $3.5 \pm 1.4$ & $2.7 \pm 0.6$ \\
\hline Inosine & $8.3 \pm 1.1$ & $32 \pm 13$ & $8.5 \pm 5.0$ & $5.5 \pm 1.4$ & $1.2 \pm 0.6$ \\
\hline$p$ value & $p<0.001$ & $p=0.02$ & $p=0.03$ & $p=0.02$ & $p<0.001$ \\
\hline
\end{tabular}

UA, uric acid; NT, nitrotyrosine; ELISA, enzyme-linked immunosorbent assay; IL, interleukin; Mac-1, macrophage-1 antigen; PCR, polymerase chain reaction; GAPDH, glyceraldehyde-3-phosphate dehydrogenase.

Secondary objectives of this study were to determine whether there are therapeutic benefits of inosine treatment in MS. Variability in disease parameters including time since initial diagnosis and disease activity during the trial, together with the relatively small subject pool, complicates such analyses. Nevertheless, while EDSS scores remained relatively constant during 6-month placebo treatment, an improvement was observed after 6 months of inosine treatment in the subjects of Arm 1. Moreover, inosine treatment resulted in a steady, statistically significant improvement in EDSS scores over a year-long period in the subjects of Arm 2. We also observed a reduction in the frequency of clinical exacerbations in patients with serum UA $>7.0 \mathrm{mg} / \mathrm{dL}$, though the difference was not statistically significant by Fisher's exact test, possibly due to a limited number of exacerbations observed.

Standardized MRI intensity values have only recently been investigated for their potential to contain MS disease-specific information. ${ }^{23}$ In this study, BP tissue regions yield distribution parameters showing high correlation with EDSS scores. The observation that the standardized PD peak location and 75th percentile values in the entire brain parenchyma simultaneously show a negative correlation with UA levels and a positive correlation with the EDSS is particularly relevant to the current study. This provides a compelling MRI indication to support the concept that the reduction in MS disability measured by EDSS that resulted from inosine treatment may be a consequence of raising serum UA levels. Larger studies are needed to confirm this observation and establish whether any of the other MRI parameters assessed may provide further insight into the effects of inosine treatment.

Studies in patients with MS and in experimental allergic encephalomyelitis (EAE), an animal model of MS, have identified a number of inflammatory markers that are modulated during active disease (for review $\mathrm{see}^{27,28}$ ). Extensive variability in these markers was seen in individuals during the placebo phase of Arm 1. Consequently, it is not surprising that significant alterations in the levels of these markers were not seen between baseline, placebo, and inosine phases. Only 5 patients in Arm 1, where there were multiple samples from placebo and inosine phases for analysis, completed the study. Nevertheless, one of these individuals exhibited a significant association between raised serum UA levels, lessened disease activity, a decrease in levels of mRNA specific for the macrophage-associated marker Mac-1 in circulating cells, and lowered levels of the peroxynitrite-dependent radical product nitrotyrosine. This decrease in nitrotyrosine levels is consistent with previous observation of nitrotyrosine clearance from the central nervous system tissue of mice with EAE that are undergoing treatment with UA. ${ }^{10}$ In addition, we detected evidence of increased Th2 activity in the peripheral blood of this individual that has previously been reported as beneficial in patients with $\mathrm{MS}^{29}$

Mechanistically, the principal therapeutic effects of inosine treatment are likely through raising serum UA levels, thereby reducing the pathological effects of peroxynitritedependent radicals. Radical-mediated oxidative stress may lead to the neuronal and oligodendrocyte loss that underlies the degenerative component of MS. In addition to findings with patients with MS, ${ }^{12,13,30}$ studies in Parkinson's disease have demonstrated that patients with higher urate levels had a slower rate of progression. ${ }^{31}$

\section{Conclusions}

Our data suggest that raising serum UA levels by the oral administration of inosine treatment may benefit patients with MS. Improvements in EDSS and a reduction in lesion activity were noted. Studies in a larger cohort are indicated and will help to elucidate the mechanistic effects of the treatment.

\section{Acknowledgments}

This work was supported by a grant from Commonwealth of Pennsylvania Department of Health to Biotechnology Foundation Laboratories (to H.K.) and grant from NIH/ NCAM (AT 001301) to D.C.H. We thank Dr. Francisco Gonzalez-Scarano for the help organizing this trial.

\section{Disclosure Statement}

No conflicts of interest exist for any of the authors listed in this article.

\section{References}

1. Morrow TJ. The costs and consequences of multiple sclerosis relapses: A managed care perspective. J Neurol Sci 2007; 256(suppl):S39-S44.

2. Noseworthy JH, Lucchinetti C, Rodriguez M, Weinshenker BG. Multiple sclerosis. NEJM 2000;343:938-952.

3. Pittock SJ, Lucchinetti CF. The pathology of MS: New insights and potential clinical applications. Neurologist 2007; 13:45-56.

4. Prat E, Martin R. The immunopathogenesis of multiple sclerosis. J Rehabil Res Dev 2002;39:187-199.

5. Neema M, Stankiewicz J, Arora A, et al. MRI in multiple sclerosis: What's inside the toolbox? Neurotherapeutics 2007;4:602-617.

6. Filippi M, Horsfield MA, Hajnal JV, et al. Quantitative assessment of magnetic resonance imaging lesion load in multiple sclerosis. J Neurol Neurosurg Psychiatry 1998; 64(suppl):S88-S93. 
7. Bagasra O, Michaels FH, Zheng YM, et al. Activation of the inducible form of nitric oxide synthase in the brains of patients with multiple sclerosis. Proc Natl Acad Sci U S A 1995;92:12041-12045.

8. Ischiropoulos $\mathrm{H}, \mathrm{Zhu} \mathrm{L}, \mathrm{Chen} \mathrm{J}$, et al. Peroxynitrite-mediated tyrosine nitration catalyzed by superoxide dismutase. Arch Biochem Biophys 1992;298:431-437.

9. Beckman JS, Ye YZ, Anderson PG, et al. Extensive nitration of protein tyrosines in human atherosclerosis detected by immunohistochemistry. Biol Chem Hoppe Seyler 1994;375:8188.

10. Hooper DC, Scott GS, Zborek A, et al. Uric acid, a peroxynitrite scavenger, inhibits CNS inflammation, blood-CNS barrier permeability changes, and tissue damage in a mouse model of multiple sclerosis. FASEB J 2000;14:691-698.

11. Kean RB, Spitsin SV, Mikheeva T, et al. The peroxynitrite scavenger uric acid prevents inflammatory cell invasion into the CNS in experimental allergic encephalomyelitis through maintenance of blood-CNS barrier integrity. J Immunol 2000;165:6511-6518.

12. Hooper DC, Spitsin S, Kean RB, et al. Uric acid, a natural scavenger of peroxynitrite, in experimental allergic encephalomyelitis and multiple sclerosis. Proc Natl Acad Sci U S A 1998;95:675-680.

13. Spitsin S, Hooper DC, Leist $\mathrm{T}$, et al. Inactivation of peroxynitrite in multiple sclerosis patients after oral administration of inosine may suggest possible approaches to therapy of the disease. Mult Scler 2001;7:313-319.

14. Starling RD, Trappe TA, Short KR, et al. Effect of inosine supplementation on aerobic and anaerobic cycling performance. Med Sci Sports Exerc 1996;28:1193-1198.

15. Dragan I, Baroga M, Eremia N, Georgescu E. Studies regarding some effects of inosine in elite weightlifters. Romanian J Physiol 1993;30:47-50.

16. Williams MH, Kreider RB, Hunter DW, et al. Effect of inosine supplementation on 3-mile treadmill run performance and VO2 peak. Med Sci Sports Exerc 1990;22:517-522.

17. Toncev G. Therapeutic value of serum uric acid levels increasing in the treatment of multiple sclerosis. Vojnosanitetski Pregled 2006;63:879-882.

18. Schulz KF. The quest for unbiased research: Randomized clinical trials and the CONSORT reporting guidelines. Ann Neurol 1997;41:569-573.

19. Ge Y, Grossman, RI, Udupa JK, et al. Magnetization transfer ratio histogram analysis of gray matter in relapsing remitting multiple sclerosis. Am J Neuroradiol 2001;22:470-475.

20. Catalaa I, Grossman RI, Kolson D, et al. Multiple sclerosis: Magnetization transfer histogram analysis of segmented normal appearing white matter. Radiology 2000;216:351-355.
21. Madabhushi A, Udupa JK, Souza A. Generalized scale: Theory, algorithms, and application to image inhomogeneity correction. Computer Vision Image Understanding 2006; 101:100-121.

22. Udupa J, Samarasekera S. Fuzzy connectedness and object definition: Theory, algorithms, and applications in image segmentation. Graphical Models Image Processing 1996;58: 246-261.

23. Lei T, Udupa JK, Moonis G, Balcer L. Hierarchical brain tissue segmentation and its application in Alzheimer's disease (AD) and multiple sclerosis (MS). SPIE Proc 2005;5746:592-603.

24. Spitsin S, Portocarrero C, Phares TW, et al. Early blood-brain barrier permeability in cerebella of PLSJL mice immunized with myelin basic protein. J Neuroimmunol 2008;196:8-15.

25. Spitsin S, Scott GS, Mikheeva T, et al. Comparison of uric acid and ascorbic acid in protection against EAE. Free Radic Biol Med 2002;33:1363-1371.

26. Enomoto A, Kimura H, Chairoungdua A, et al. Molecular identification of a renal urate anion exchanger that regulates blood urate levels. Nature 2002;417:447-452.

27. Olsson T. Critical influences of the cytokine orchestration on the outcome of myelin antigen-specific T-cell autoimmunity in experimental autoimmune encephalomyelitis and multiple sclerosis. Immunol Rev 1995;144:245-268.

28. Eng LF, Ghirnikar RS, Lee YL. Inflammation in EAE: Role of chemokine/cytokine expression by resident and infiltrating cells. Neurochem Res 1996;21:511-525.

29. Mouzaki A, Deraos S, Chatzantoni K. Advances in the treatment of autoimmune diseases; cellular activity, type-1/ type-2 cytokine secretion patterns and their modulation by therapeutic peptides. Curr Medicinal Chemistry 2005;12: 1537-1550.

30. Spitsin S, Hooper DC, Mikheeva T, Koprowski H. Uric acid levels in patients with multiple sclerosis: Analysis in monoand dizygotic twins. Multiple Sclerosis 2001;7:165-166.

31. Schwarzschild MA, Schwid SR, Marek K, et al. Serum urate as a predictor of clinical and radiographic progression in Parkinson disease. Arch Neurol 2008;65:716-723.

Address reprint requests to: Hilary Koprowski, M.D.

Biotechnology Foundation Laboratories at Thomas Jefferson University 1020 Locust Street

JAH, Room M-85

Philadelphia, PA, 19107

E-mail: hilary.koprowski@jefferson.edu 
